\title{
Computing Partition Functions of PCFGs
}

\author{
Mark-Jan Nederhof • Giorgio Satta
}

\section{Erratum to: Res Lang Comput (2008) 6: 139-162 DOI: 10.1007/s11168-008-9052-8}

The following acknowledgment had been unfortunately omitted from the text when the authors submitted the final version.

Acknowledgments We gratefully acknowledge fruitful discussions with Kousha Etessami and Mihalis Yannakakis on issues directly related to this article and with Luca Bergamaschi and Luigi Grippo on issues concerning the solution of nonlinear systems of equations. Much of this research was done while the first author was affiliated to the Research Group on Mathematical Linguistics, Rovira i Virgili University, supported by a grant from the Spanish Ministerio de Educación y Ciencia, SAB2004-0044.

The online version of the original article can be found under doi:10.1007/s11168-008-9052-8.

M.-J. Nederhof $(\bowtie)$

School of Computer Science, University of St Andrews, North Haugh,

St Andrews KY16 9SX, Scotland, UK

e-mail: markjan.nederhof@gmail.com

G. Satta

Department of Information Engineering, University of Padua, Via Gradenigo 6/A,

35131 Padova, Italy

e-mail: satta@dei.unipd.it 